\section{Usefulness of Maalox \\ for Detection of the Precise \\ Bleeding Points and \\ Confirmation of Hemostasis on Gastrointestinal Hemorrhage}

For endoscopists, gastrointestinal hemorrhage is one of the major occurrences encountered in general practice as a lifethreatening emergency. The first goal is to stop the bleeding endoscopically without surgical treatment [1], because hypovolemic hypotension and delay in hemostasis increase the surgical risk and adversely affect the patient's survival [2].

Improvements in endoscopic diagnosis and techniques have increased the opportunity to treat early esophageal, gastric, and colorectal cancer endoscopically. However, the major complications of endoscopic treatments are bleeding and perforation [3]. Unsuccessful endoscopic attempts to overcome such complications need surgical rescue.

One of the keys to establish hemostasis in both situations is to identify the precise bleeding points. Maalox (Rohrer, Fort Washington, Pennsylvania, USA), an aluminium hydroxide-magnesium hydroxide combination liquid drug, is one of the widely used antacids, which is easily administered from the instrumentation channel of the endoscope. Maalox contrasts blood, and it is common to apply staining techniques to improve endoscopic diagnosis in the gastrointestinal tract [4]. Before administration, it is often difficult to precisely identify the bleeding points because blood clots stain the ulcer bed red. Once Maalox is poured on, the reddish ulcer bed changes to white, and then several reddish streams of blood emerge from behind the Maalox. As this method of detecting bleeding points resembles condensed milk being poured on strawberries, we call this phenomenon the "strawberrymilk sign." A positive strawberry-milk sign means continuous hemorrhage and the strawberry-milk sign is negative when the ulcer bed remains white after hemostasis. We report here the usefulness of Maalox by showing a patient who had an artificial ulcer with oozing bleeding caused by endoscopic mucosal resection (Figure 1)

\section{Fujishiro, H. Ono, T. Gotoda,}

H. Yamaguchi, H. Kondo, D. Saito

Endoscopy Division, National Cancer

Center Hospital, Tokyo, Japan
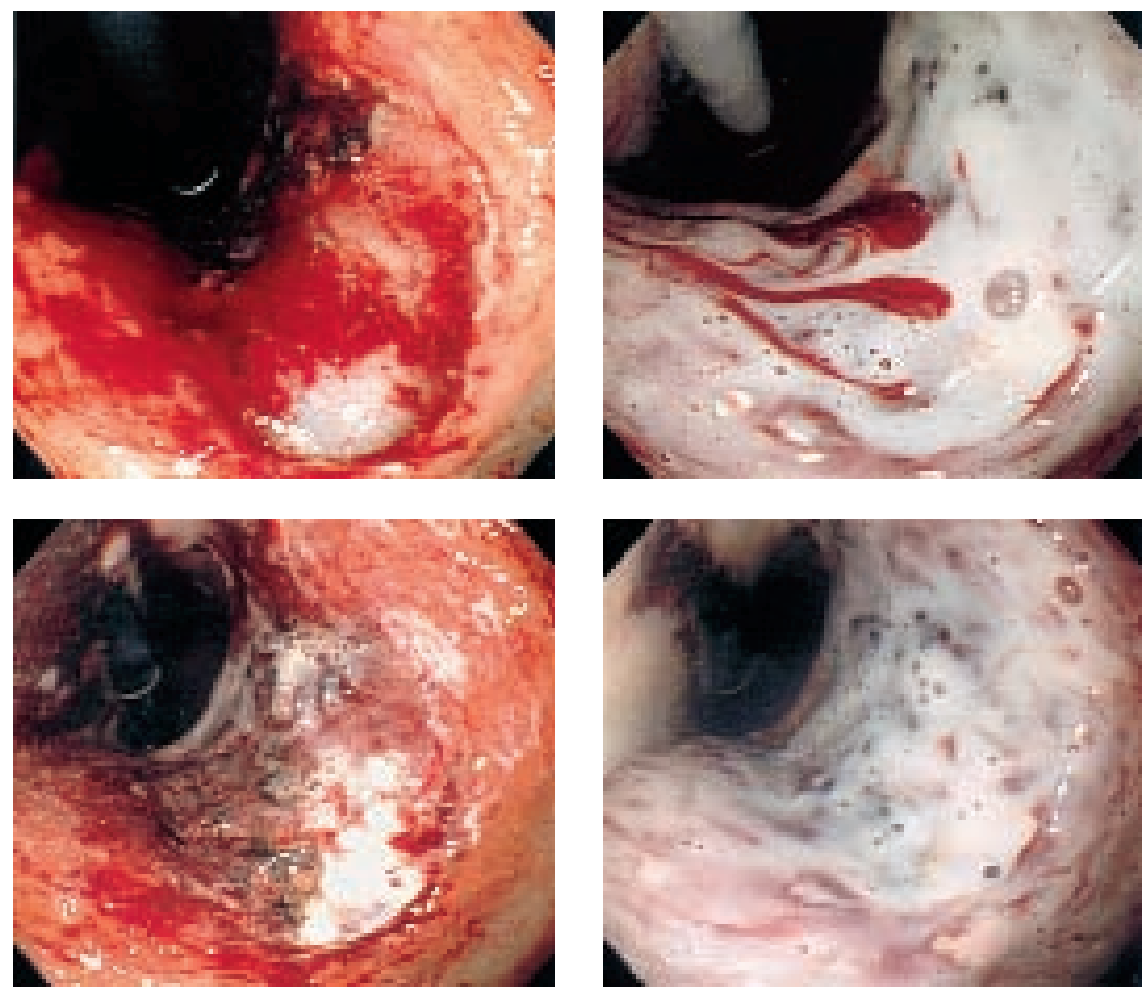

Figure 1 The patient experienced bleeding after endoscopic mucosal resection. a The resected area shows oozing blood. b Three bleeding points were detected after Maalox was poured on (strawberry-milk sign was positive).

\section{References}

${ }^{1}$ Cook DJ, Guyatt H, Salena BJ, et al. Endoscopic therapy for acute nonvariceal upper gastrointestinal hemorrhage: a meta-analysis. Gastroenterology 1992; 102: $139-148$

${ }^{2}$ Lau JY, Sung JJ, Lam YH, et al. Endoscopic retreatment compared with surgery in patients with recurrent bleeding after initial endoscopic control of bleeding ulcers. N Engl J Med 1999; 340: $751-756$

${ }^{3}$ Inoue H. Endoscopic mucosal resection for esophageal and gastric mucosal cancers. Can J Gastroenterol 1998; 12: $355-359$ c Hemostasis was achieved by the use of thermocoagulation. d No bleeding points were detected after Maalox was poured on (strawberry-milk sign was negative)

${ }^{4}$ Shim CS. Staining in gastrointestinal endoscopy: clinical application and limitation. Endoscopy 1999; 31: 487-496

Corresponding Author

H. Ono, M.D.

Endoscopy Division

National Cancer Center Hospital

5-1-1 Tsukiji

Chuo-ku

Tokyo

Japan

Fax: $\quad+81-3-35423815$

E-mail: hyono@gan2.ncc.go.jp 